\title{
Minimal Model for Sand Dunes
}

\author{
Klaus Kroy*, Gerd Sauermann, and Hans J. Herrmann \\ PMMH, École Sup. de Physique et Chimie Industrielles. 10, rue Vauquelin, 75231 Paris, Cedex 05, France.
}

\begin{abstract}
We propose a minimal model for aeolian sand dunes. It combines an analytical description of the turbulent wind velocity field above the dune with a continuum saltation model that allows for saturation transients in the sand flux. The model provides a qualitative understanding of important features of real dunes, such as their longitudinal shape and aspect ratio, the formation of a slip face, the breaking of scale invariance, and the existence of a minimum dune size.
\end{abstract}

Sand dunes develop wherever sand is exposed to an agitating medium (air, water...) that lifts grains from the ground and entrains them into a surface flow. The diverse conditions of wind and of sand supply in different regions on Earth give rise to a large variety of shapes of aeolian dunes [1] 3]. Moreover, dunes have been found on the sea-bottom and even on Mars. Despite the long history of the subject, the underlying physical mechanisms of dune formation are still not very well understood. How are aerodynamics (hydrodynamics) and the particular properties of granular matter acting together to create dunes? How is the shape of a dune maintained when it moves? In the following we propose a "minimal model" for aeolian sand dunes to address such questions. Although it refers only to rather generic properties of the wind velocity field and the laws of aeolian sand transport, it can make interesting qualitative predictions that are not sensitive to the simplifying assumptions, e.g. about the surface profile, the development and position of the slip face, dune migration etc. Using results from turbulent boundary layer calculations [4] [- we will propose an approximate analytical description of the surface shear stress exerted by the wind onto a heap of sand. This will be combined with a saltation model [7] that allows for saturation transients of the sand flux, which are an essential element of a consistent description of dunes.

We start by summarizing some basic knowledge about aeolian sand transport and saltation. The mean turbulent wind velocity above a plane surface increases logarithmically with height. Its magnitude is specified by a characteristic velocity called the "shear velocity" $u_{*}$ and defined by $u_{*}^{2} \equiv \tau_{0} / \rho$ with $\tau_{0}$ the average surface shear stress (far away from any obstacle) and $\rho$ the density of air. On a surface covered with sand, the wind entrains some grains into a surface layer flow if the shear velocity exceeds a threshold value. The grains advance mainly by an irregular hopping process, thereby reducing the wind velocity in the surface layer. A unique relation between the shear stress $\tau$ and the sand flux $q$ is thus established in the equilibrium state. If $\tau$ is not too close to the threshold, one has approximately [1, 2, 8, 9]

$$
q_{s} \propto \tau^{3 / 2} .
$$

The index $s$ emphasizes that this simple relation is restricted to situations where the flux is saturated. According to Eq.(11), the changing wind shear stress above a heap of sand $h(x, y)$ is responsible for flux gradients, which cause erosion and deposition. Due to mass conservation, the flux gradient $d q / d x$ on a slice $h(x)$ parallel to the wind direction gives rise to a change in height with time $d h / d t$, and thus to migration of the surface profile in the wind direction with a velocity

$$
v \propto d q / d h \text {. }
$$

This velocity is much smaller than the velocity of the saltating grains, so that the terrain can be assumed to be stationary for considerations concerning the wind and saltation dynamics. To obtain $q_{s}$ and the corresponding $v_{s} \equiv v\left(q_{s}\right)$ from Eqs.(1) and (2), we thus need to know the stationary surface shear stress perturbation $\hat{\tau} \equiv \tau / \tau_{0}-1$ caused by the heap. This depends on its shape.

In a first attempt to model $\hat{\tau}$, one might try the affine relation $\hat{\tau} \propto h$ and combine it with the saturated flux approximation $q=q_{s}$. If this was true, the surface velocity $v$ would increase with height $(d v / d h \geq 0)$ due to the nonlinearity of Eq.(1). This in turn would decrease the upwind slope and increase the lee slope, and thus eventually create a slip face on the lee side, where sand slides down in avalanches. Since there is so far nothing in the model to stop the further decrease of the upwind slope, our model "dune" would then start to decrease in height and finally become flat, whatever its windward profile. This "zeroth order model" is obviously missing an essential ingredient. In the following, we will successively demonstrate how the modeling of both the shear stress and the flux have to be refined to achieve a consistent description of dune formation and migration. In particular, we will show that a subtle balance of two quantitatively small but qualitatively crucial corrections to the zeroth order model determine the shape of aeolian heaps and

*present address: Physics Dept., University of Edinburgh, King's Buildings, Edinburgh EH9 3JZ, Scotland 


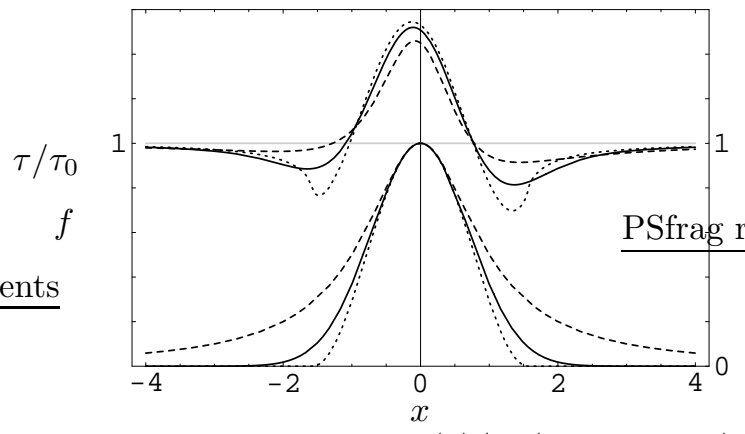

FIG. 1. Surface shear stress $\tau(x) / \tau_{0}$ (upper curves) above the profiles of Eq. (5) (lower curves: $f_{L}$ dashed, $f_{G}$ solid, $f_{C}^{2}$ dotted line) according to Eq.(4) with $\alpha H / L=0.45, \beta=0.25$. The small windward shift of the maximum shear stress with respect to the apex is crucial for dune formation.

dunes.

An improved description for the shear stress can be obtained from turbulent boundary layer calculations. The surface shear stress perturbation $\hat{\tau}_{x}$ in wind direction caused by a smooth bump $h(x, y)$ is [5, 6]

$$
\mathcal{F}_{x y}\left\{\hat{\tau}_{x}\right\}=\frac{\alpha k_{x}\left(k_{x}+i \beta\left|k_{x}\right|\right)}{\left(k_{x}^{2}+k_{y}^{2}\right)^{1 / 2}} \mathcal{F}_{x y}\{h(x, y)\} .
$$

Here, we have neglected a minor term $\propto \ln \left|k_{x} L\right|$, and abbreviated the Fourier transformation from $x, y$ to $k_{x}, k_{y}$ by $\mathcal{F}_{x y}$. The parameters $\alpha$ and $\beta$ depend logarithmically on the ratio $L / z_{0}$, where $L$ is the characteristic length of $h(x, 0)$ and $z_{0}$ is a measure of the surface roughness [10]. For the following discussion we evaluate Eq.(3) on a slice $h(x) \equiv h(x, 0)$ of a transverse dune ( $h$ independent of $y)$. We obtain $\left(h^{\prime}:=d h / d x\right)$

$$
\hat{\tau}(x)=\alpha\left[\int_{-\infty}^{\infty} d \xi \frac{h^{\prime}(x-\xi)}{\pi \xi}+\beta h^{\prime}(x)\right] .
$$

Minor corrections for dunes with a finite transverse width can be calculated [1] but are omitted here for simplicity.

For certain heap profiles $h(x) \equiv H f(x / L)$ (Fig. 1) with

$$
f_{L}=\left(1+x^{2}\right)^{-1}, f_{G}=e^{-x^{2}}, f_{C}^{n}=\cos ^{n} x,
$$

Eq.(4) can be evaluated analytically. (The cosine profile $f_{C}^{n}$ is understood to vanish outside the region $[-\pi / 2, \pi / 2]$.) The plots of Eq.(14) in Fig. 1 share several crucial properties not present in the affine approximation $\hat{\tau} \propto h$. First, we observe from a dimensional analysis of Eq.(4) that $\hat{\tau} \propto H / L$ for a given shape $f$. Hence, as one expects from the scale invariance of turbulence, the amplification of the shear velocity at the top of the profile is determined by its aspect ratio $H / L$ and is essentially independent of the absolute height $H$ [10]. Secondly, at the tails of the profiles in Fig. 11, the shear stress decreases below its asymptotic value $\tau_{0}$. This effect is particularly pronounced for the profile $f_{C}^{n}$ that has a discontinuous second derivative. Further, as a consequence of the second term in Eq.(4), the surface shear stress is asymmetric

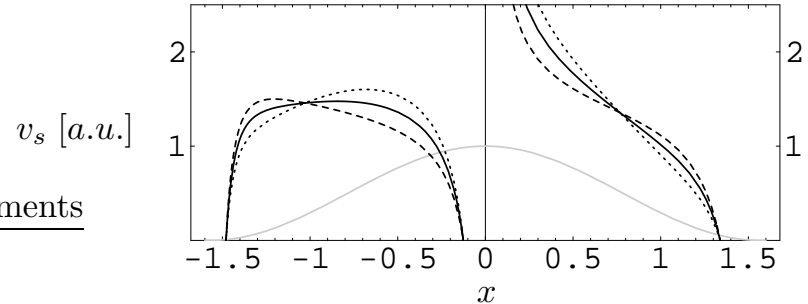

FIG. 2. The surface velocity $v_{s}(x)$ of the cosine-shaped heap $f_{C}^{2}(x)$ (gray) according to Eqs.(1), (2), (4) for $\alpha=3.2$, $\beta=0.25$, and varying aspect ratios $H / L=0.01$ (dashed), 0.14 (solid), 0.27 (dotted). Note the tendency to increase the (unstable) lee slope and to drive the windward slope towards a (stable) optimum value that depends on $\alpha$ and $\beta$. If $f_{C}^{2}$ is interpreted as the envelope of a dune and its separation bubble, the slip face must be located near the sharp drop of $v_{s}(x)$ slightly upwind from the top of the envelope.

even for symmetric profiles like those of Eq.(5). For $q=q_{s}$, there can thus be a net deposition on a symmetric heap of sand and the shift of the shear stress maximum with respect to the top of the heap [12] allows deposition at the top of the heap. An initially flat heap of sand can spontaneously grow and increase its windward slope.

To understand this instability of a plane sand surface, the migration velocity $v_{s}(x)$ of a cosine shaped heap of sand $f_{C}^{2}(x)$ is depicted in Fig. 2. The decrease of $v_{s}(x)$ on the lee side reveals the anticipated self-amplifying instability of the lee slope, which gives rise to the formation of the slip face. More interestingly, Eq. (4) renders $v_{s}(x)$ approximately constant over almost the whole windward side if $H / L$ is close to a stable optimum value. Slightly better results can be achieved for slightly lower $n$ (with slightly larger $H / L$ ) but not for the profiles $f_{G}$ and $f_{L}$, for which $v_{s}$ is always non-uniform. The dashed and dotted lines in Fig. 2 were obtained for a smaller and a larger aspect ratio and represent a steepening $\left(v_{s}^{\prime}<0\right)$ and flattening $\left(v_{s}^{\prime}>0\right)$ of the windward side, respectively. Altogether, Fig. 2 suggests that the coupled Eqs.(1) and (4) drive a heap of sand towards a "dune" with a cosinelike windward profile of a preferred aspect ratio, and a slip face on the lee side.

From the preceding qualitative analysis it is not yet obvious that this process converges to a translation invariant steady state solution. Several previous studies using similar descriptions either did not scrutinize the long time behavior of their models 13, 14, or failed to obtain stable dunes [15,16. Indeed, the present model is still insufficient. The problem with the quasi-linear Eqs.(3) and (4) is that they were derived for smooth hills and cannot account for flow separation. Once a slip face has developed, with a slope of about $32^{\circ}-35^{\circ}$ that terminates in a sharp brink, they can no longer be applied. As in the textbook example of a backward facing step, 
flow separation occurs at the brink and large eddies develop in the wake region. The recirculating flow in this "separation bubble" is bounded by a separating streamline against the region of laminar flow. Following a suggestion by Zeman and Jensen [15], we assume that in order to calculate the shear stress on the windward side, Eqs.(3) and (4) may be applied to the envelope of a dune and its separation bubble (inset of Fig. 3) in place of the actual surface profile of the dune. In our numerical computations we model this effective separation bubble by a third order polynomial that is the smooth continuation of the windward dune profile with a maximum slope of $14^{\circ}$ [17.

If this generalization is accepted, and if - for qualitative purposes - one identifies the profile $f_{C}^{2}$ with such an envelope, one can draw further interesting conclusions from Fig. 2. First, the constant $v_{s}(x)$ obtained for the windward side of the cosine profiles $f_{C}^{n}(n \approx 2)$, provides strong evidence that Eqs.(1) and (14) allow a dune with a cosine-like windward side of suitable aspect ratio to remain invariant upon translation by aeolian sand transport. The steep decrease of $v_{s}(x)$ at a certain distance upwind from the top of the envelope, which is a consequence of the maximum of $\tau(x)$ being shifted with respect to the top of the envelope, must then coincide with the position of the brink. Secondly, the stable dune shape is scale invariant $\mid 10]$. Together with the scaling relation $\hat{\tau} \propto H / L$ mentioned above, this implies that the migration velocity $v$ of dunes obeys $v \propto u_{*}^{3} / L$, in accord with the empirical observation of a roughly reciprocal relation between dune velocity and dune height [1, 2].

However, one has to bear in mind that Eq.(1) is restricted to a completely saturated saltation layer. Saturation transients in the sand flux, as they occur under variable wind or sand conditions, introduce a new characteristic length, the saturation length $\ell_{s}$ [7] related to (but distinct from) the mean saltation length of the grains. Shape invariance is therefore restricted to large dunes $\left(L / \ell_{s} \rightarrow \infty\right)$. Estimates [7] of typical absolute values of $\ell_{s}$ suggest that real dunes are not necessarily large in this sense, in accord with field measurements [18] that report size dependent dune shapes. Eq.(1) also fails at boundaries sand/ground. It predicts deposition at the windward foot of an isolated dune, where the shear stress decreases 77, 19] (Fig. 2). Previous numerical studies tried to avoid this by focusing onto the short time behavior and by ad hoc heuristic methods such as a "smoothing operator" 13] or an "adaptation length" [14].

In the following, we demonstrate how these problems are naturally cured if Eq.(11) is replaced by a slightly more elaborate description derived from a continuum saltation model [7]. In the steady state $(\partial / \partial t \simeq 0)$, that model reduces to the differential equation

$$
\ell_{s} \partial q / \partial x=q\left(1-q / q_{s}\right)
$$

for the sand flux $q$, and explicit expressions for $\ell_{s}(\tau)$ and $q_{s}(\tau)$. For wind velocities well above the entrainment threshold, $\ell_{s}(\tau)$ levels off, and the saturated flux $q_{s}$

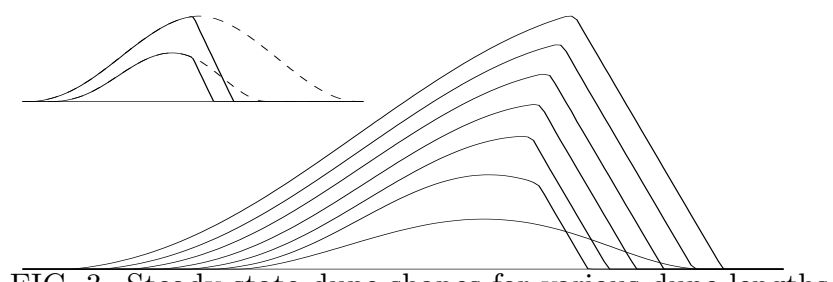

FIG. 3. Steady state dune shapes for various dune lengths (roughly $50 \ell_{s} \ldots 100 \ell_{s}$ ) obtained from Gaussian heaps by integrating Eqs.(2) and (6). Flow separation at the brink was modeled heuristically by introducing a separation bubble (dashed lines in the inset). Periodic boundary conditions were applied to conserve the sand mass and thus allow for a steady state. The influx/outflux vanishes for the dunes with slip face but is finite for the heap. The time evolution of the latter is shown in Fig $4 \mathrm{~b}$. (Plot rescaled for visualization.)

converges to Eq.(1). The detailed forms used in our numerical integrations, which involve the entrainment threshold, can be found in [7] but are not important here. During the integration of Eqs.(2) and (6), the lee slope of the heap is constantly evaluated. If it exceeds $14^{\circ}$, the separation bubble is introduced for the shear stress calculation as described above. Additionally, the surface shear stress is set to zero within the separation zone, and surface avalanches are induced if the slope exceeds the angle of repose $\left(34^{\circ}\right)$. For definiteness we choose the values $\alpha=3.2, \beta=0.25$ for the parameters of Eq.(位). Starting from a Gaussian heap, we obtain the steady state profiles shown in Fig. 3. Their general shape and average windward slope of about $10^{\circ}$ are in good accord with recent field measurements 18, 20. The shape of the largest dune (indeed well described by $f_{C}^{n}, n \approx 2$ ) nicely confirms our expectations from Fig. 2, whereas for small heaps, the instability of the lee slope is washed out by the saturation transients. The upwind shift of $\tau(x)$ with respect to $h(x)$ (which is of the order of $\beta L$ ) and the lag of $q(x)$ with respect to $\tau(x)$ and $q_{s}(x)$ (of the order of $\ell_{s}$ ) compete to establish a critical heap size for slip face formation. Smaller heaps do not spontaneously develop a slip face, while somewhat larger heaps evolve into a steady state dune with a windward slope that is lowered and a slip face that is shifted downwind compared to the asymptotic shape. The critical dune size depends on the wind velocity through (and scales as) the saturation length $\ell_{s}(\tau) \approx \ell_{s}\left(\tau_{0}\right)(\approx$ for large $\tau$ ).

Fig. 1 shows the growth histories of two heaps of different mass and initial aspect ratio $H / L$. It demonstrates the spontaneous formation of a slip face that is stable for the larger heap but finally turns out to be unstable for the smaller one. For intermediate sizes, flow separation can stabilize an existing slip face although it would not have developed from a flat configuration. This is the case for the two smallest dunes with slip face in Fig. 3, which were produced from a steep initial configuration (as in Fig. (Ab). As well as the critical heap size for slip face 
a)

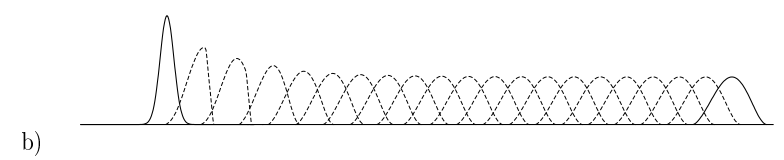

FIG. 4. Growth histories for two Gaussian heaps of different mass and initial aspect ratio (periodic boundary conditions). The steep initial configuration of the heap in part b) gives rise to the temporary formation of a slip face, which turns out to be unstable. Note that the distances to reach the steady state shape are different. (Plots rescaled for visualization.)

formation (out of a flat initial condition), there exists thus a somewhat smaller critical dune size for slip face destruction and an intermediate regime, where the presence of a slip face depends on the initial conditions. This implies, in particular, that a slip face that develops out of a flat initial condition has always a finite height. Indeed, we find that slip face destruction also occurs at a finite height.

In summary, we proposed a minimal model for aeolian sand dunes. The advantage of the simple Eqs.(3) and (4) over more elaborate nonlinear models 21 23] for the wind shear stress is that they combine a high computational efficiency with good accuracy [6], and can thus be applied to simulate large scale desert topographies. The drawback is that we had to introduce the heuristic separation bubble [17]. We pointed out that the saturation transients contained in Eq.(6) break the expected scale invariance and are responsible for the existence of a critical heap size for slip face formation and a critical dune size for slip face destruction. Further, we analyzed the mechanisms responsible for the longitudinal shape and aspect ratio of dunes and heaps, and for slip face formation. It is an interesting question whether (and how) these results can be generalized to full $3 d$ problems. The deviations of the wind velocity from the main wind direction are small above an obstacle that is flat in the transverse direction. One can thus expect that the present approach applies to transverse dunes and, with

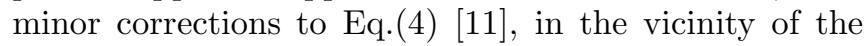
symmetry plane of a crescent-shaped barchan dune. As a direct consequence of our above results, only $3 d$ heaps larger than a certain minimum size can spontaneously develop a slip face, which then has a finite height and width. This minimum size should scale as $\ell_{s}$, which is a decreasing function of $u_{*}$ [7]. Preliminary results from an extension of the model to $3 d$ support this picture.
We gratefully acknowledge financial support by the DFG (HE 2732/1-1), and helpful discussions with J. Soares Andrade Jr. and M. E. Cates.

[1] R. A. Bagnold, The physics of blown sand and desert dunes. (Methuen, London, 1941).

[2] K. Pye and H. Tsoar, Aeolian sand and sand dunes (Unwin Hyman, London, 1990).

[3] N. Lancaster, Geomorphology of desert dunes (Routledge, London, 1995).

[4] P. S. Jackson and J. C. R. Hunt, Q. J. R. Meteorol. Soc. 101, 929 (1975).

[5] J. C. R. Hunt, S. Leibovich, and K. J. Richards, Q. J. R. Meteorol. Soc. 114, 1435 (1988).

[6] W. S. Weng et al., Acta Mech. Suppl. 2, 1 (1991).

[7] G. Sauermann, K. Kroy, and H. J. Herrmann, Phys. Rev. E 64, 031305 (2001).

[8] R. S. Anderson and P. K. Haff, Science 241, 820 (1988).

[9] Aeolian Grain Transport 1 \& 2, Acta Mechanica Suppl., edited by O. E. Barndorff-Nielsen and B. B. Willetts (Springer, Wien, New York, 1991).

[10] As long as $L$ and $z_{0}$ do not change by orders of magnitude, this weak logarithmic dependence is negligible.

[11] Analytic evaluation for a dune with a Gaussian transverse profile of width $\sigma$ shows that the main effect of a finite transverse width is to renormalize $\beta \rightarrow \beta(\sigma) \lesssim \beta$ and to add a (trivial) term $\gamma(\sigma) h(x)$ with $\gamma(\infty)=0$ and $\gamma(L / \sqrt{2}) \approx-0.2$ within the brackets of Eq.(四).

[12] For the contour $f_{L}$, the relative shift $\delta x / L$ of the maximum of $\tau(x)$ with respect to the maximum of $f(x)$ is $2\left(1+\beta^{2}\right)^{1 / 2} \sin [\arctan (\beta) / 3]-\beta \approx-\beta / 3(\beta \ll 1)$.

[13] F. K. Wippermann and G. Gross, Boundary Layer Meteorology 36, 319 (1986).

[14] P. M. van Dijk, S. M. Arens, and J. H. van Boxel, Earth Surf. Process. Landforms 24, 319 (1999).

[15] O. Zeman and N. O. Jensen, Ris $\varnothing$ National Laboratory M-2738, (1988).

[16] J. M. T. Stam, Sedimentology 44, 127 (1997).

[17] We have carefully tested this heuristic procedure against $2 d$ and $3 d$ numerical solutions from the finite volume solver FLUENT5 (Fluent Inc. 1999) and verified that the predictions for the windward side are reasonably stable against the freedom thus introduced into the model.

[18] G. Sauermann, P. Rognon, A. Poliakov, and H. J. Herrmann, Geomorphology 36, 47 (2000).

[19] G. F. S. Wiggs, I. Livingstone, and A. Warren, Geomorphology 17, 29 (1996).

[20] P. A. Hesp and K. Hastings, Geomorphology 22, 193 (1998).

[21] R. I. Sykes, J. Fluid Mech. 101, 647 (1980).

[22] O. Zeman and N. O. Jensen, Q. J. R. Meteorol. Soc. 113, 55 (1987).

[23] J. H. van Boxel, S. M. Arens, and P. M. van Dijk, Earth Surf. Process. Landforms 24, 255 (1999). 